\title{
Pneumatic tools for vitreoretinal surgery
}

This article was published in the following Dove Press journal:

Clinical Ophthalmology

10 March 2012

Number of times this article has been viewed

\section{Mario R Romano Jose Luis Vallejo-Garcia Alessandro Randazzo Paolo Vinciguerra}

Istituto Clinico Humanitas, Via Manzoni 56, 20089 Rozzano, Milan, Italy
Correspondence: Mario R Romano Istituto Clinico Humanitas, Via Manzoni 56, 20089, Rozzano, Milan, Italy

Tel +390282 244680

$\mathrm{Fax}+390282244690$

Email romanomario@email.it
Abstract: One of the difficulties of microsurgery is learning how to control physiological tremors. The pneumatic tool eliminates the physiological tremor, but no tactile feedback is provided. The manual tremor when closing the forceps is completely eliminated and the exact target can be more easily grabbed. Forceps closure pressure can rise up to 50 psi, whereas the scissors can be used in two modes: multicut and proportional. When performing bimanual surgery the pedal range is divided into two steps: in the first step, the forceps are controlled, and in the second step, the forceps remain closed. At the same time the scissors start to work in the preselected mode. No adverse events occurred and no iatrogenic retinal breaks were produced. Precision and control sensation were a grateful surprise.

Keywords: pneumatic forceps, pneumatic scissors, physiological tremor

\section{Introduction}

Since Machemer's work in his garage in 1970, where he developed a 17-gauge single port multifunctional vitrectome, vitreoretinal surgery has achieved an extremely high technological progression. ${ }^{1}$ The development of multiple accessories like forceps, scissors, and endolaser probes led to the popularization of vitrectomy. ${ }^{2}$ This encouraged surgeons to undergo more complex cases, as vitrectomy became safer, with better results. ${ }^{3}$ The difficulties of microsurgery are learning how to control physiological tremor. The pneumatic tool eliminates the physiological tremor, theoretically simplifying microsurgical procedures, but no tactile feedback is provided. We present our initial surgical experience with these pneumatic tools in 15 macular surgeries.

\section{Methods}

\section{Study design}

We conducted a prospective interventional case series on 15 eyes of 15 consecutive patients, diagnosed with epiretinal membranes (ERM; ten eyes), full thickness macular holes (FTMH; three eyes) and diabetic macular tractional retinal detachments (TRD; two eyes).

\section{Subjects}

To be eligible for enrollment, the patients had to have functional impairment as well as a diagnosis of ERM or FTMH or macular TRD confirmed by spectral domain optical coherence tomography (SD-OCT). We excluded patients younger than 18 years of age, with severe systemic disease, pregnant women, any uncontrolled ocular disease, and/or myopia $\geq 6$ diopters. 


\section{Examinations}

Pre- and postoperative examinations included a detailed ophthalmic history, refracted best corrected visual acuity (BCVA; Sloan early treatment diabetic retinopathy study vision charts), slit lamp biomicroscopy, Goldman tonometry, and SD-OCT. The study protocol included a minimum of four clinical visits: baseline, day of surgery, and at 1 and 3 postoperative months.

\section{Interventions}

Cataract surgery was performed in all cases 1 week before the vitrectomy. The surgical procedure included a 25-gauge three-port pars plana vitrectomy and ERMinternal limiting membrane (ILM) peeling assisted with blue dye. The peeling was performed with ILM pneumatic forceps (seven cases) and Maxgrip pneumatic forceps (six cases) (Alcon-Grieshaber, Fribourg, Switzerland).

The TRD cases were performed with bimanual maxgrip forceps and curved scissors. The vitreous base was thoroughly trimmed during vitrectomy. The retinal periphery was inspected for retinal breaks. In TRD cases, $360^{\circ}$ endolaser photocoagulation was performed. A fluid-air exchange was then performed with humidified air. Prone positioning was advised after surgery for FTMH and TRD eyes.

\section{Outcome measures}

The primary endpoint was to describe the use of new pneumatic forceps and to report any adverse events that may have occurred during or after the surgery.

Secondary outcomes were changes in best-corrected visual acuity and damage to the outer retinal layer (ORL) in the area where the peeling was started. The peeling was started in the extrafoveal area located infero-temporal to the macula. The ORL damage was defined as an interruption of external limiting membrane (ELM) or inner-outer segment (IS/OS) junction visible on the OCT-scan.

\section{Results}

Safety profile was confirmed; neither intraoperative nor postoperative retinal breaks were found. No damage or contusion to the internal retinal layers was produced during the surgery. No interruption of ELM or IS/OS junction was visible on OCT-scan in the area where the peeling was started (Figure 1). The learning curve was very straightforward, achieving full control during the first surgery. The precision was considered better by the surgeon.
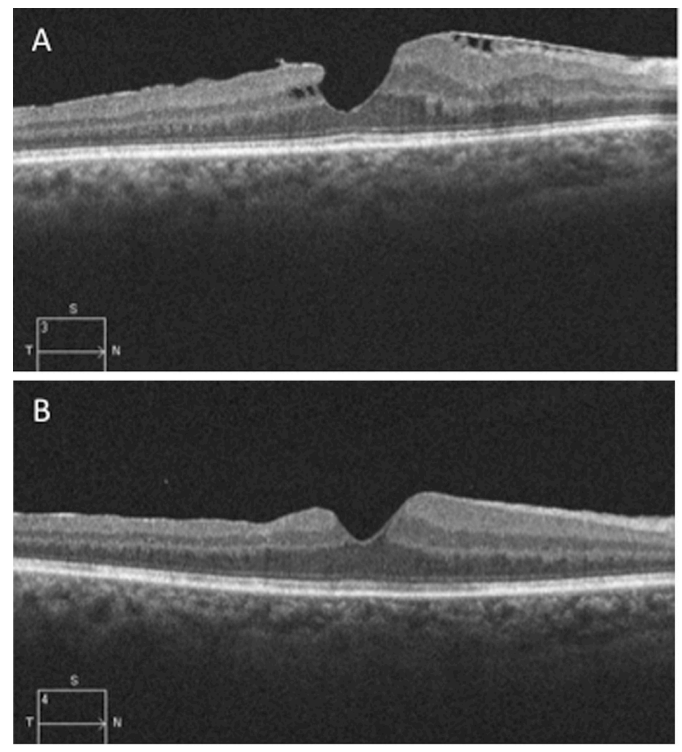

Figure I The preoperative OCT scan showed a tractional epiretinal membrane with intraretinal cysts.

Notes: (A) Three months after surgery the scan demonstrated a release of inner traction and resolution of cysts (B) No visible damage of outer retinal layer. Abbreviation: OCT, optical coherence tomography.

The best set up of the pressure values for the forceps was found to start with 5 psi with a maximum pressure of $30 \mathrm{psi}$, which was enough in order to provide a consistent grasp.

In the TRD, bimanual surgery was realized with both pneumatic tools: the Maxgrip forceps and curved scissors. The scissors were used in multicut and proportional cut and achieved 450 cuts per minute. The initial control is not so intuitive, but the surgeon gets used to the bimanual performance during the first surgery.

When performing bimanual surgery the pedal range is divided in two steps: with the first one the forceps are controlled and when we go on to the second step, the forceps maintain closure, and at the same time the scissors start to work in the preselected mode, multicut or proportional.

The preoperative BCVA was analyzed in all groups. In the ERM group (10 eyes) the mean BCVA before the surgery was 0.46 (SD 0.13) with a range $0.25-0.65$. There was a significant improvement in VA $(P=0.0003)$ with a postoperative mean BCVA of 0.72 (SD 0.17) and a range of 0.5-1.0. In the macular hole group (3 eyes), presurgical mean BCVA was 0.12 (SD 0.07) with a range of 0.05-0.2. No significant improvement was achieved $(P=0.07)$ as postoperative mean BCVA improved to 0.25 (SD 0.2), range $0.2-0.4$. In the TRD group the mean BCVA before the surgery was 0.2 (SD 0.2). There was a significant improvement in VA $(P=0.02)$ with a postoperative mean BCVA of 0.35 (SD 0.1) at last follow-up. 


\section{Conclusion}

The potential iatrogenic damage (retinal holes, ORL damage, choroidal neovascularization) is located in the area where the surgeon performs the first grab of the membrane and from which, once the flap is lifted, the surgeon will start the peeling. ${ }^{4-6}$ One of the main difficulties of microsurgery is to learn how to control physiological tremor. Pneumatic accessories, like forceps and scissors, have been developed by AlconGrieshaber. Although they don't offer anything different as tip forceps, they are an important step in precision and control of the tip. The manual tremor experienced when closing the forceps is completely eliminated and the exact target can be more easily grabbed. Opening and closing pressure of the tip are regulated with the vitrectomy pedal, allowing customization of the range of parameters in a personal fashion. All different Grieshaber tips (Alcon-Grieshaber, Fribourg, Switzerland) are available (ILM forceps, end-grasping forceps, asymmetrical forceps, Maxgrip forceps) and also pneumatic curved and vertical scissors. Forceps closure pressure can rise up to $50 \mathrm{psi}$, but we found from $5 \mathrm{psi}$ at the beginning of the foot pedal to $30 \mathrm{psi}$ at the end of foot pedal adequate. Scissors can be used in two modes, multicut and proportional. The multicut achieves a frequency of 450 cuts per minute, while the proportional is a straightforward cut. Multicut mode is very useful when cutting extensive proliferative membranes, reducing considerably the time employed for segmentation and improving greatly its safety. The tip's tremor is completely eliminated, therefore iatrogenic retinal breaks are less frequently produced.
The first five surgeries were all pucker peelings. We strongly advise implementing this pathology, as it is easier to get the new grabbing feeling with tougher membranes. We were surprised by the comfortable and rapid segmentation of the proliferative tissue; full control of the scissor tip guarantees the advantage of pneumatic tools compared to manual scissors. The learning curve and adaptation was achieved quickly to pneumatic instrumentation. The only disadvantage is cost as they are disposable precise instruments. We are currently conducting a comparative study evaluating the retinal sensitivity changes induced by pneumatic versus manual forceps in the peeled area.

\section{Disclosure}

The authors report no conflicts of interest.

\section{References}

1. Machemer R, Buettner H, Norton EW, Parel JM. Vitrectomy: a pars plana approach. Trans Am Acad Ophthalmol Otolaryngol 1971;75(4):813-820.

2. Machemer R, Buettner H, Parel JM. Vitrectomy, a pars plana approach. Instrumentation. Mod Probl Ophthalmol 1972;10:172-177.

3. Machemer R, Norton EW. Vitrectomy, a pars plana approach. II. Clinical experience. Mod Probl Ophthalmol 1972;10:178-185.

4. Sandali O, El Sanharawi M, Basli E, et al. Paracentral retinal holes occurring after macular surgery: incidence, clinical features, and evolution. Graefes Arch Clin Exp Ophthalmol. 2012 Jan 27. [Epub ahead of print].

5. Mason JO 3rd, Feist RM, Albert MA Jr. Eccentric macular holes aftervitrectomy with peeling of epimacular proliferation. Retina. 2007;27:45-48.

6. Natarajan S, Mehta HB, Mahapatra SK, Sharma S. A rare case of choroidal neovascularization following macular hole surgery. Graefes Arch Clin Exp Ophthalmol. 2006;244:271-273.
Clinical Ophthalmology

\section{Publish your work in this journal}

Clinical Ophthalmology is an international, peer-reviewed journal covering all subspecialties within ophthalmology. Key topics include: Optometry; Visual science; Pharmacology and drug therapy in eye diseases; Basic Sciences; Primary and Secondary eye care; Patient Safety and Quality of Care Improvements. This journal is indexed on

\section{Dovepress}

PubMed Central and CAS, and is the official journal of The Society of Clinical Ophthalmology (SCO). The manuscript management system is completely online and includes a very quick and fair peer-review system, which is all easy to use. Visit http://www.dovepress.com/ testimonials.php to read real quotes from published authors. 